\title{
Methodology Report \\ Use of BODIPY (493/503) to Visualize Intramuscular Lipid Droplets in Skeletal Muscle
}

\author{
Espen E. Spangenburg, ${ }^{1}$ Stephen J. P. Pratt, ${ }^{2}$ \\ Lindsay $M$. Wohlers, ${ }^{1}$ and Richard M. Lovering ${ }^{2}$ \\ ${ }^{1}$ Department of Kinesiology, University of Maryland, School of Public Health, College Park, MD 20742, USA \\ ${ }^{2}$ Department of Orthopaedics, University of Maryland, School of Medicine, Baltimore, MD 21201, USA
}

Correspondence should be addressed to Richard M. Lovering, rlovering@som.umaryland.edu

Received 1 May 2011; Revised 21 July 2011; Accepted 2 August 2011

Academic Editor: Robert J. Bloch

Copyright ( 2011 Espen E. Spangenburg et al. This is an open access article distributed under the Creative Commons Attribution License, which permits unrestricted use, distribution, and reproduction in any medium, provided the original work is properly cited.

\begin{abstract}
Triglyceride storage is altered across various chronic health conditions necessitating various techniques to visualize and quantify lipid droplets (LDs). Here, we describe the utilization of the BODIPY (493/503) dye in skeletal muscle as a means to analyze LDs. We found that the dye was a convenient and simple approach to visualize LDs in both sectioned skeletal muscle and cultured adult single fibers. Furthermore, the dye was effective in both fixed and nonfixed cells, and the staining seemed unaffected by permeabilization. We believe that the use of the BODIPY (493/503) dye is an acceptable alternative and, under certain conditions, a simpler method for visualizing LDs stored within skeletal muscle.
\end{abstract}

\section{Introduction}

Triacylglycerol (aka triglyceride, or TAG) storage is typically at its highest appreciable amounts in white adipose tissue. TAGs are stored within the organelles termed neutral lipid droplets (LDs). Previous studies have shown that under various conditions, appreciable LD accumulation can also occur in other tissues, such as skeletal muscle. The appearance of LDs in nonadipose depots is often referred to as "ectopic fat". Increasing amounts of ectopic fat are associated with a number of clinical conditions, such as peripheral insulin resistance and muscular dystrophies [1-3]. Thus, the ability to measure or visualize the accumulation of stored TAG in the form of LDs in skeletal muscle is important.

Measurement of lipid accumulation within skeletal muscle has been performed using numerous different methodologies, each with their own strengths and weaknesses. Total TAG concentration can be quantified through biochemical extraction, magnetic spectroscopy, or visually analyzed with Oil Red O (ORO) or Nile Red histochemical staining of tissue sections [4]. Each approach has unique advantages and disadvantages. The use of ORO has become the most commonly used approach to visualize LDs within a section of skeletal muscle [4-7]. Since LD frequency is correlated with the oxidative potential of the muscle fiber, there often is an uneven distribution of fibers that contain a significant amount of LDs within a whole muscle. Specifically, Van Loon et al. demonstrated that LD frequency is significantly higher in type I fibers compared to type II fibers, albeit there was no distinction between the subtypes of the type II fibers in this study [8]. Thus, it is often necessary to consider the amount of LDs in a fiber-by-fiber approach. Staining of the fibers allows the investigator to consider multiple labeling approaches for colocalization studies. This approach precludes experimental bias from extracellular TAG that is often found in skeletal muscle, but not within the muscle fiber itself.

The use of ORO as a means to measure LDs in a fiberspecific manner is a very powerful and useful technique for muscle biologists. However, the use of ORO is not without experimental disadvantages. For example, the tissue of interest requires fixation, preventing its use on live cells. In addition, completely dissolving ORO into solution can be challenging. It is typically dissolved in isopropanol, may require heating to encourage the process, and must be filtered afterwards, which often reduces the final concentration of 
the solution. Once in solution, the ORO has a limited shelf life, thus requiring the solution to be remade frequently. Finally, the ORO staining process requires the handling and disposal of a significant amount of hazardous chemicals. For a number of years, adipocyte biologists have used an alternative approach to ORO to visualize LDs within adipocytes, specifically a cell permeable lipophilic fluorescence dye, BODIPY (493/503), which emits bright green fluorescence [9]. The dye stains neutral LDs in live or fixed cells and can be successfully coupled with other staining and/or labeling approaches. An advantage of the dye is that it requires little effort to place into solution and, unlike ORO, does not need to be made "fresh" for each use.

The purpose of this paper is to demonstrate the use of BODIPY (493/503) to image LDs within skeletal muscle fibers as an alternative to ORO. In order to determine its flexibility of use, we examined the use of BODIPY (493/503) under a variety of conditions that included frozen muscle and freshly cultured single muscle fibers.

\section{Methods}

2.1. Animals. A variety of skeletal muscles were removed from male Sprague-Dawley rats $(\sim 275 \mathrm{~g})$ and male C57BL/ $10 \mathrm{ScSn}$ mice $(\sim 32 \mathrm{~g})$ while under inhalation anesthesia $(\sim 3-$ $5 \%$ isoflurane via a nosecone using a precision vaporizer, cat \# 91103, Vet Equip, Inc, Pleasanton, Calif, USA) just prior to sacrifice. All animal procedures were approved by the Animal Care and Use Committee at the University of Maryland.

2.2. Muscle Fiber Sections. Rat tibialis anterior (TA) muscles were isolated and frozen in liquid nitrogen-cooled isopentane. They were stored at $-80^{\circ} \mathrm{C}$, and transverse sections were cut on a cryostat ( $10 \mu \mathrm{m}$ thick) and collected onto glass slides (Superfrost Plus; VWR, Westchester, Pa, USA).

2.3. Single Fiber Isolation. Intact single muscle fibers were isolated from the flexor digitorium brevis (FDB) muscle of adult mice according to previously described techniques [10]. Briefly, surgically excised FDB muscles were incubated in dissociation media (DM) containing DMEM, $1 \mu \mathrm{L} / \mathrm{mL}$ gentamycin, $0.2 \% \mathrm{BSA}$, and $4 \mathrm{mg} / \mathrm{mL}$ of collagenase A (Sigma, C0130). FDB muscles were placed in a $35 \mathrm{~mm}$ dish with $4 \mathrm{mLs} \mathrm{DM}$ and then in an incubator $\left(37^{\circ}, 5 \% \mathrm{CO}_{2}\right)$ for 2 hours. Following the dissociation, muscles were placed in a new $35 \mathrm{~mm}$ plate with fresh media and triturated with a small bore $(\sim 1 \mathrm{~mm})$ fire polished glass transfer pipette to yield single FDB myofibers. Following the trituration process, fibers were plated onto extracellular matrix- (ECM, Sigma E1270) coated dishes (Matek, P35G-1.0-14-C). Fibers were allowed to incubate overnight before staining and/or immunolabeling. Some dishes were incubated overnight in a high-fat media containing equal molar concentrations of palmitate (PA) and oleate (OA) $(0.5 \mathrm{mM}$ of each thus a final concentration $1.0 \mathrm{mM}$ ) to induce LD formation in the fibers. The palmitate and oleate were conjugated to $2 \%$ fatty acidfree bovine serum albumin which would be expected to result in a final ratio of NEFA to BSA of 2.5:1.0, as previously described [11]. We have found no evidence of toxicity when cells are treated with both palmitate and oleate, although we have found evidence of toxicity if cells are treated with palmitate alone.

2.4. BODIPY (493/503) Staining and Visualization. BODIPY (D3922, Molecular Probes, Carlsbad, Calif, USA) (excitation wavelength $480 \mathrm{~nm}$, emission maximum $515 \mathrm{~nm}$ ), was diluted in PBS or DMSO at a concentration of $1 \mathrm{mg} / \mathrm{mL}$ and applied to cross sections or FDB fibers, respectively, for 30 mins. We assessed both fixed (4\% paraformaldehyde for 5 mins) and unfixed sections, as well as fixed (4\% paraformaldehyde for 5 mins) and live FDB fibers; 4,6-diamidino-2-phenylindole (DAPI) was used to identify nuclei. Following fixation, samples were washed 3 times in phosphate-buffered saline (PBS) for 10 mins. Some cross sections were stained with $5 \mathrm{ug} / \mathrm{mL}$ rhodamine-labeled succinylated wheat germ agglutinin (WGA, RL1022S; Vector Laboratories, Burlingame, Calif, USA), and some were labeled with antibodies (monoclonal slow myosin, M4276, Sigma, St Louis, Mo, USA) to identify slow (Type I) muscle fibers. For immunolabeling, sections were washed for 10 minutes in $100 \mathrm{mmol}$ glycine phosphate-buffered saline (glycine PBS), blocked for 1 hour in 1\% bovine serum albumin PBS (BSA-PBS), and then incubated for 2 hours with primary antibodies diluted to $2 \mathrm{mg} / \mathrm{mL}$ in PBS. The tissue sections were then washed 3 times with $1 \%$ BSA/PBS for 10 minutes before incubation with species-specific secondary antibodies coupled to Alexa dye 568 (Invitrogen, Carlsbad, Calif, USA) (dilution of $1: 100)$. All samples were mounted in VECTASHIELD (Vector Laboratories) and covered with glass cover slips (No. 1, VWR). We examined samples under epifluorescent optics, and digital images were obtained with a Zeiss 510 confocal laser scanning microscope.

2.5. Oil Red O Staining. Serial sections $(10 \mu \mathrm{M})$ from frozen rat TA muscle were used. The first half of the serial sections were stained with BODIPY as described above. The remaining samples were air dried onto a glass slide and then fixed in formalin for 5 minutes, followed by a 1 minute wash with tap water. Next, slides were rinsed with $60 \%$ isopropanol then stained with a filtered 5\% Oil Red O solution for 30 minutes. After Oil Red O staining, slides were rinsed with $60 \%$ isopropanol. All samples were mounted in VECTASHIELD (Vector Laboratories), covered with glass cover slips (No. 1, VWR), and mounted with Permount (Biomeda, Burlingame, Calif, USA).

\section{Results}

BODIPY (493/503) has become the standard dye utilized by scientists to study LDs within adipocytes. In this study, we sought to demonstrate that this compound can be used to visualize LDs in skeletal muscle tissue. Using the BODIPY (493/503), we were able to detect visible LDs through standard epifluorescence and confocal microscopy in sections of whole rat muscle and live cultured mouse single fibers. Using cross sections of a soleus muscle taken 


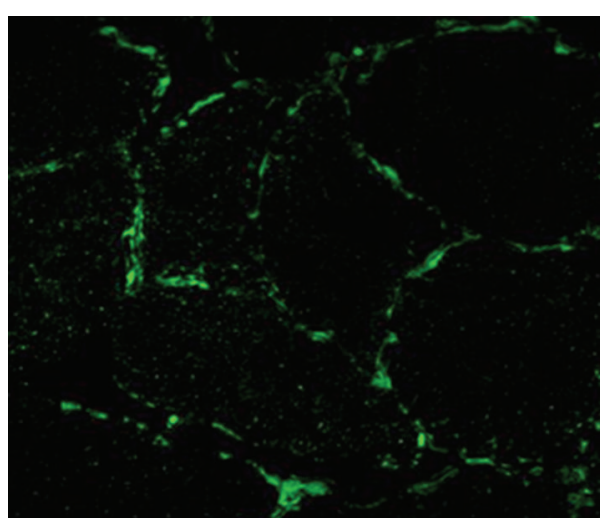

(a)

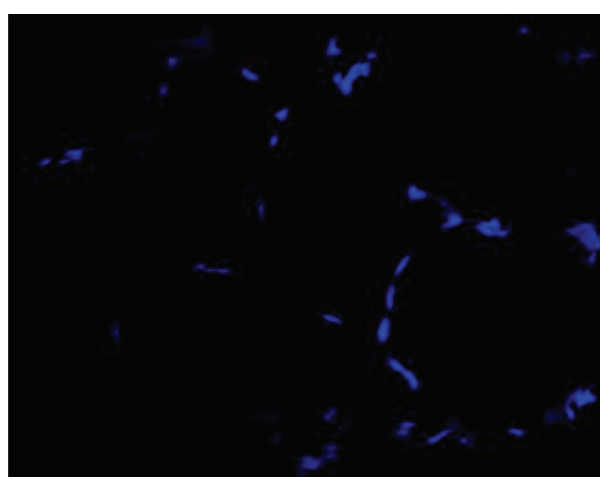

(c)

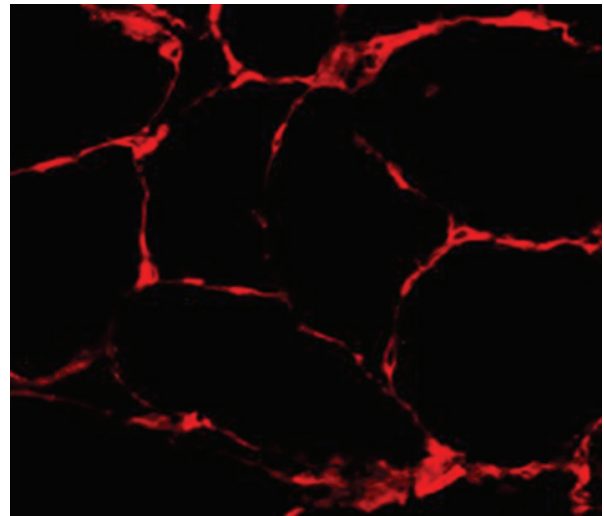

(b)

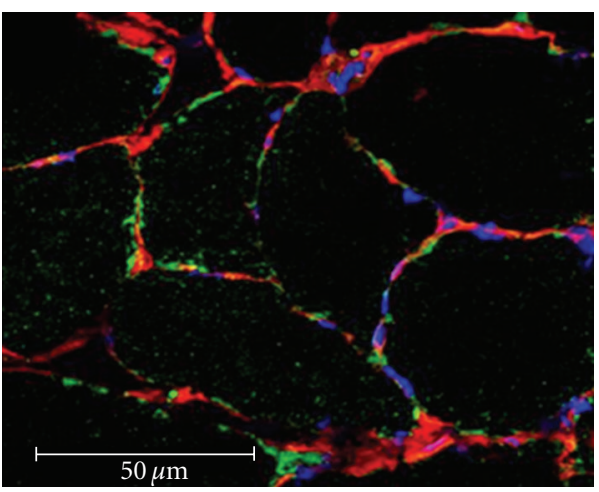

(d)

FIGURE 1: Staining of neutral lipid droplets (LDs) with BODIPY (493/503) in cross section of rat soleus muscle demonstrates staining within the fiber, but especially under the surrounding sarcolemma. (a) Fibers stained with BODIPY (green) demonstrate the appearance of spherical LDs within the muscle fibers as previously described with Oil Red O staining procedures. (b) To provide anatomical localization, the membrane is labeled with wheat germ agglutinin (WGA)-Alexa Fluor 594 (red). (c) Nuclei were stained with DAPI (Blue). (d) Merged triple label image of the muscle section. All images were taken at $63 \mathrm{x}$, and a scale bar is provided for reference.

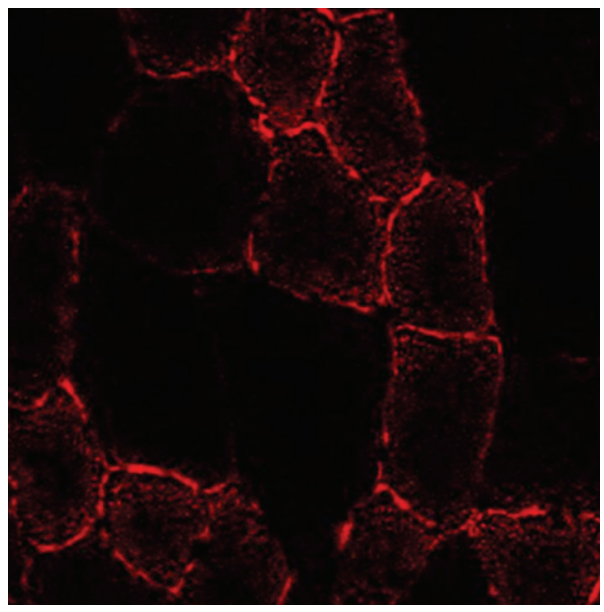

(a)

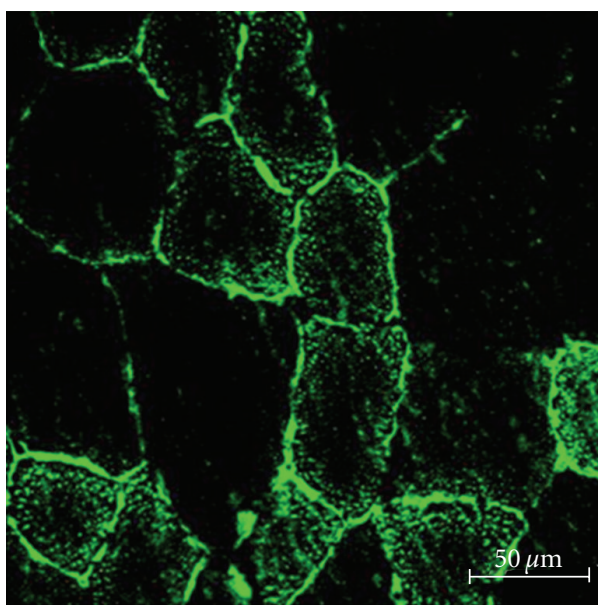

(b)

FIGURE 2: Comparison of serial cross sections from rat tibialis anterior (TA) muscle demonstrates that ORO and BODIPY staining are the strongest in the same fibers. (a) Fibers stained with ORO (red) demonstrate the appearance of spherical LDs within the muscle fibers. (b) Fibers stained with BODIPY (green) demonstrate the appearance of spherical LDs within the muscle fibers. 


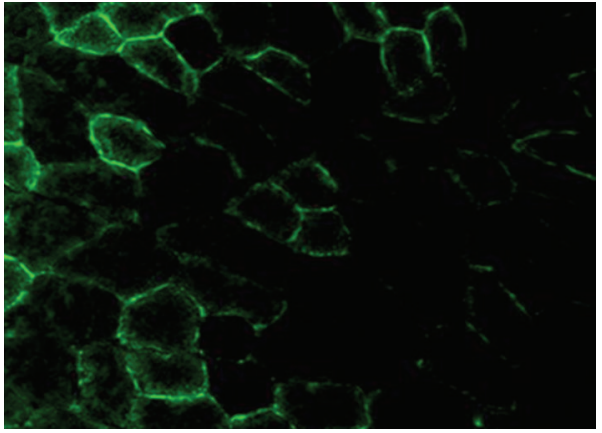

(a)

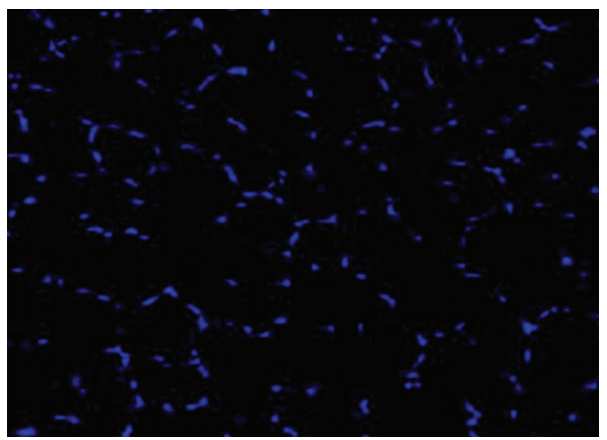

(c)

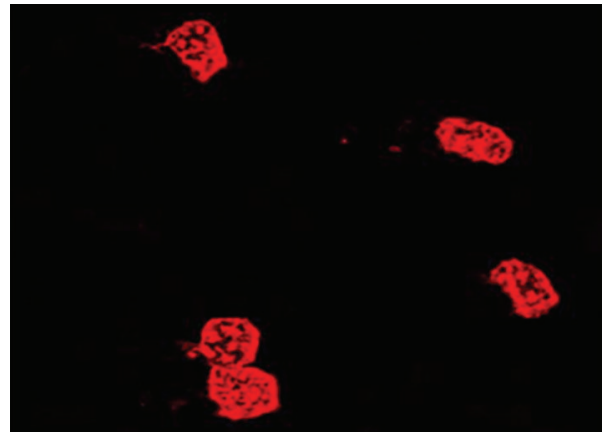

(b)

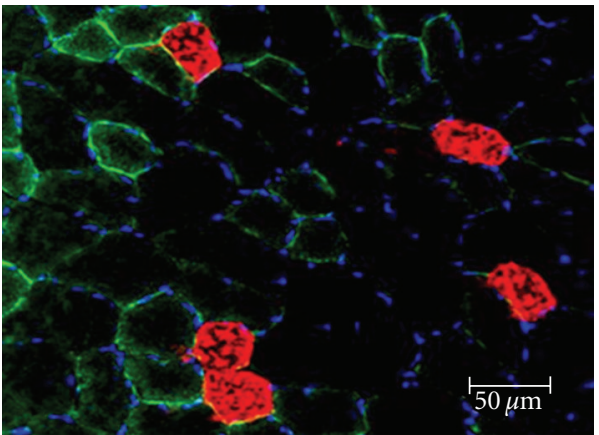

(d)

FIGURE 3: Staining of LDs with BODIPY (493/503) in cross section of rat tibialis anterior (TA) muscle demonstrates that LDs are not limited to muscle fibers expressing slow MHC. (a) Fibers stained with BODIPY (green) demonstrate the appearance of spherical LDs within the muscle fibers as previously described with Oil Red O staining procedures. (b) Slow fibers were labeled using an antibody specific for the slow form of MHC (red). (c) Nuclei were stained with DAPI (Blue). (d) Merged triple label image of the muscle section demonstrates fibers presenting with substantial BODIPY signal that do not label for slow MHC. All images were taken at 10x, and a scale bar is provided for reference.

from a rat, we found visible LDs (green, Figure 1(a)) situated within the muscle and along the sarcolemma, identified by wheat germ agglutinin staining (red). These staining patterns are very similar to our ORO images (Figures 2(a) and 2(b)) and also to previously published ORO images taken from muscle cross sections [6, 7].

Previous publications have suggested that LDs are typically more abundant in the type I MHC-positive fibers $[2,12]$. Thus, we attempted to determine if LDs stained by BODIPY (493/503, green) were selectively found in only type I muscle fibers (red, Figures 3 and 4). Surprisingly, we found with regular frequency that a number of fibers that contained substantial LD stain (bright fibers in Figures 3 and 4) were not necessarily type I (Figure 3 ). To explore this in greater detail, we examined additional sections at increased magnification. We found that LDs are clearly not restricted to type I fibers, but are also abundant in fast fibers (presumably type IIa) (Figure 4).

To assess the use of BODIPY in live cells, we isolated and cultured single muscle fibers from the FDB of adult mice. Fibers were either placed in standard culture condition media (Figures 5(a) and 5(c)) or placed in media supplemented with equimolar concentrations of fatty acids (Figures 5(b) and 5(d)). The fibers were stained with BODIPY (493/503) and DAPI (blue). In the fibers maintained under standard culture conditions, we found a heterogeneous mix of fibers with easily detectable LDs (Figure 5(a)) and fibers with virtually no detectable LDs (Figure 5(c)). However, when the fibers were treated overnight with the $1.0 \mathrm{mM}$ FA mixture, the majority of the fibers exhibited an obvious increase in the amount of visible LDs that were found throughout the entire length of all fibers (Figures 5(b) and 5(d)). We have detected increases in LD frequency across a range of 0.5$1.0 \mathrm{mM}$ FA in cultured cells (data not shown). Furthermore, using 3D confocal reconstruction of the treated fibers, it was clear that LDs were located throughout the volume of the FAtreated fibers. In agreement with previous publications, the presented Z-stack images ( $1 \mu \mathrm{M}$ thick, Figure 6 ) allow for the appreciation of the size of the LD within the muscle fiber. The LDs in muscle are quite small $(<2 \mu \mathrm{M})$, particularly when compared to what is found in cultured adipocytes $(>10 \mu \mathrm{M})$ or even LDs within hepatic tissue $(\sim 10 \mu \mathrm{M})$.

\section{Discussion}

The purpose of this paper is to demonstrate the use of BODIPY (493/503) as an alternative approach to image LDs within skeletal muscle fibers. Currently, the most common approach to visualize LDs in skeletal muscle is through ORO staining, which requires tissue fixation and is a more timeconsuming process. From an experimental point of view, BODIPY is a more flexible approach when compared to 


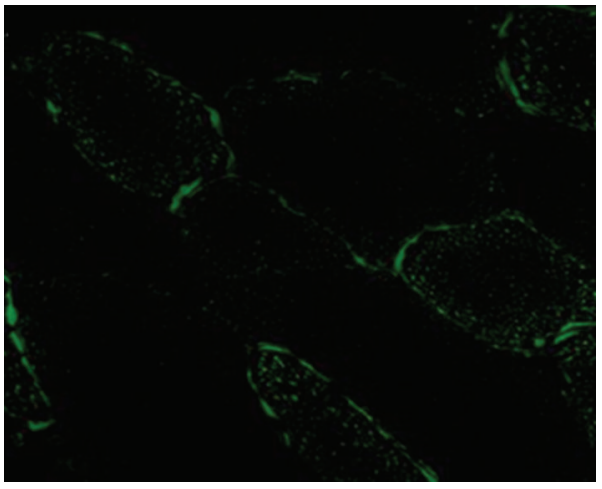

(a)

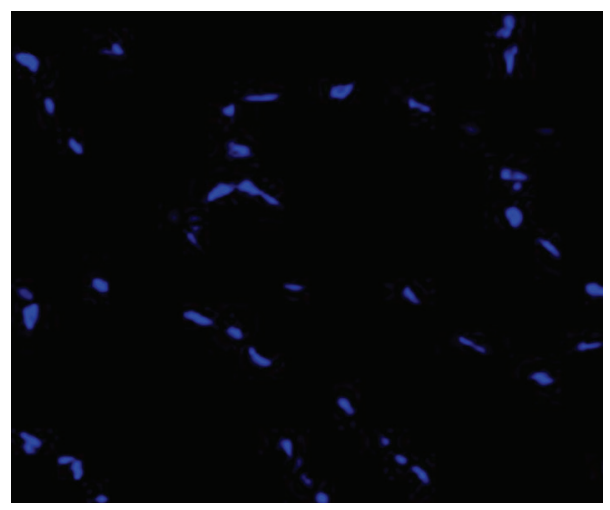

(c)

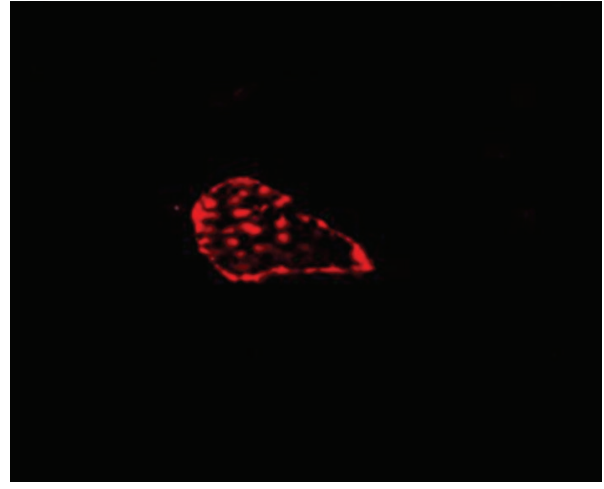

(b)

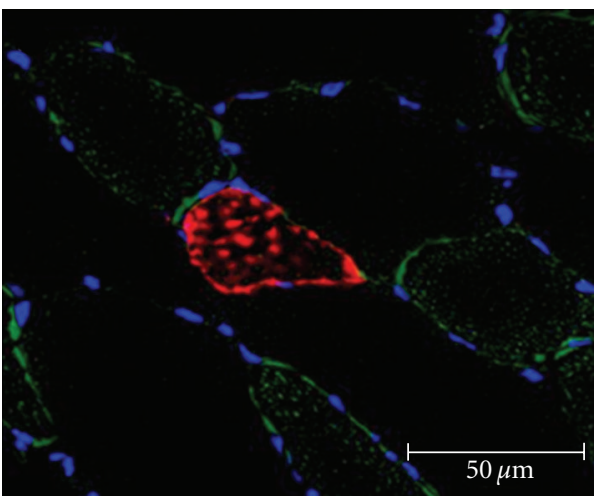

(d)

FIgURE 4: High-magnification image demonstrates the presence of LDs within nonslow muscle fibers in the cross section of the rat TA. (a) Fibers stained with BODIPY (green) demonstrate the appearance of spherical lipid droplets within the muscle fibers. (b) Slow fibers were identified using an antibody specific for the slow form of MHC (red). (c) Nuclei were stained with DAPI (Blue). (d) Merged triple label image of the muscle section demonstrates fibers presenting with substantial BODIPY signal that do not label for slow MHC. All images were taken at $63 \mathrm{x}$, and a scale bar is provided for reference.

ORO. Specifically, we were able to use BODIPY on live cells and fixed cells with no appreciable differences. We also found that permeabilization did not affect staining, although others have noted that saponin treatment can reduce the number of LDs [13]. We also used BODIPY on fixed and unfixed frozen muscle sections with equal success. We found that ORO and BODIPY (493/503) resulted in comparable results between serial cross sections of the same muscle, indicating that BODIPY $(493 / 503)$ is an effective alternative for LD staining in muscle. To date, only a few studies have used BODIPY as a means for visualizing LDs in muscle. Prats et al. used BODIPY (493/503) to show very clear staining of LDs in the soleus muscle [13], and, in a more recent paper, Kostominova used BODIPY to identify LDs in extraocular and peripheral muscle $[14,15]$. Unfortunately, these papers have not resulted in increased utilization of this approach in skeletal muscle. Thus, for whatever reason, we believe muscle biologists may not be aware of BODIPY (493/503) as a means for examining LD presence in skeletal muscle. To the best of our knowledge, our current work represents the first use of BODIPY to identify LDs in fast-twitch skeletal muscle isolated myofibers and whole-muscle cross sections. Our images clearly demonstrate the ease with which LDs can be visualized using the BODIPY stain in various experimental approaches in skeletal muscle.

A number of fluorescent dyes can be employed to visualize LD within skeletal muscle. ORO and Nile Red have both been successfully used in skeletal muscle [5-7]; however, each has limitations that reduce their functionality in a laboratory setting. For example, Nile Red is difficult to use in experiments that require multiple labeling, because it emits a powerful signal spanning a large wavelength [9]. ORO has been used with more regularity; however, since ORO is dependent on the use of isopropanol, it can cause the artificial fusion of LDs, making it inappropriate for analysis of LD size or number [9]. Furthermore, ORO must be filtered; tissue requires fixation and can be difficult dissolve. Scientists that study LD function in nonmuscle cells have adopted the use of BODIPY (493/503) as a means to visualize LDs within cells. BODIPY (493/503) contains a nonpolar structure that, upon binding to neutral lipid, emits a green fluorescence signal with a narrow wavelength range, making it an ideal approach for using in multilabeling (or dye) experiments [9]. The hydrophobic nature of these dye molecules promotes rapid entry into the nonpolar environment of LDs [9]. Gocze and Freeman showed that the 


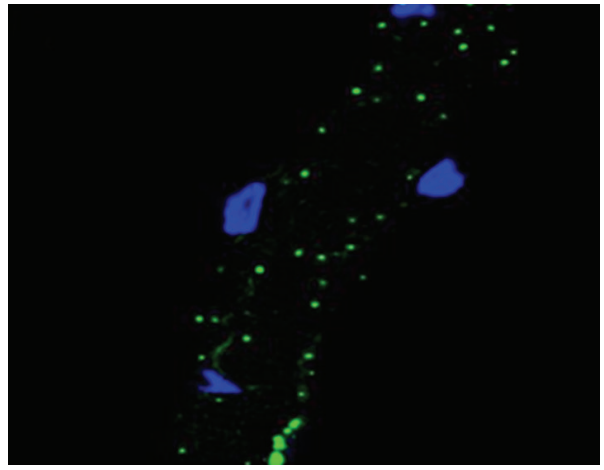

(a)

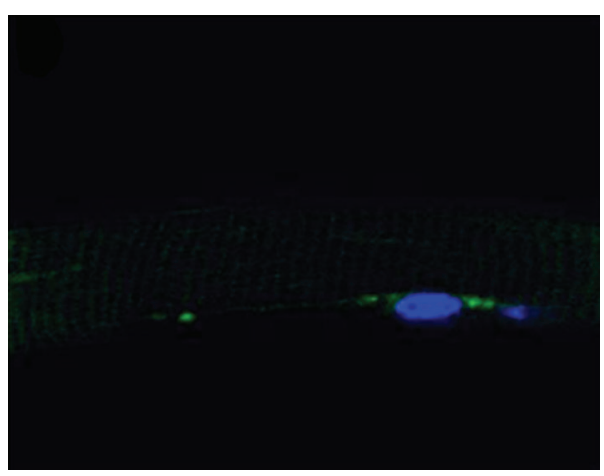

(c)

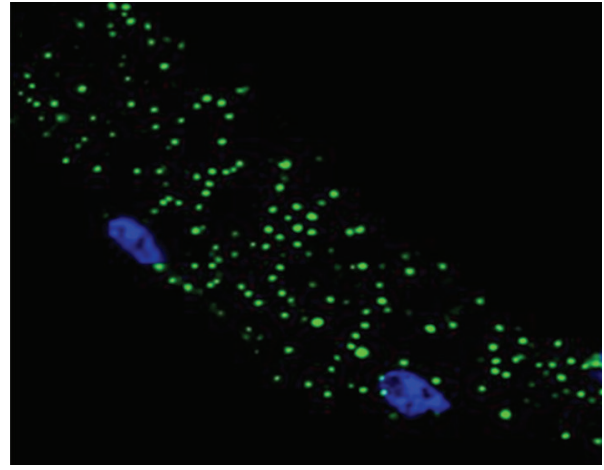

(b)

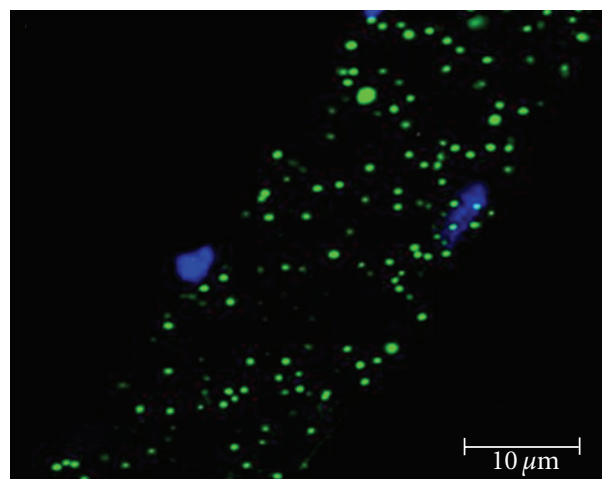

(d)

FIGURE 5: Exposure of single fibers to a palmitate-oleate mixture substantially increases the size and frequency of LDs within cultured mouse single muscle fibers. (a, c) Single muscle fibers isolated from the FDB were doubly stained with BODIPY (493/503-green) and DAPI (blue). The fibers were cultured overnight in standard media conditions. A heterogeneous mix of fibers presenting with LDs was found, in which some fibers presented with a visible number of LDs (a), while others presented with virtually no LDs (c). (b, d) Single muscle fibers isolated from the FDB were cultured overnight in standard media conditions supplemented with albumin-conjugated palmitate-oleate mixture ( $0.5 \mathrm{mM}: 0.5 \mathrm{mM}$ ratio). A substantially higher number and larger LDs were found consistently across all fibers that were imaged. All images were taken at $63 \mathrm{x}$, and a scale bar is provided for reference.

lipid fluorescent variability is significantly lower when using BODIPY (493/503) compared to Nile Red, suggesting that it may be more specific for the LD [16].

Here, we visually detected the appearance of spherical LDs within individual skeletal muscle fibers consistent with previous descriptions [6]. In muscle cross sections, the LDs were often in close proximity to the sarcolemma, which suggests that sarcolemmal identification may be critical to determine if the LDs are located in intramuscular or intermuscular regions. This would be particularly critical if the goal is quantification of LDs within muscle fibers. LDs were found scattered throughout the individual fibers (in some, but not all) in a punctuate fashion. The punctuate pattern and the inability to find LDs in all fibers agree with previous descriptions and our findings of LD distribution based on ORO staining of muscle cross sections [6]. We also found that when staining isolated fibers in standard cell culture media, LDs were not consistently visible in all of the fibers within the culture dish. These data suggest that LDs are not found in great frequency across all fibers. However, when we treated the isolated single fibers with media containing high concentrations of fatty acids, we found a substantial increase in the frequency of LDs and found LD present within all fibers. As expected, we found that BODIPY (493/503) works remarkably well in multiple-labeling approaches using both sectioned muscle and cultured single fibers. For example, we found that muscle fibers that contained a higher frequency of LDs were not always positive for type I MHC. Previously, van Loon et al. demonstrated a high correlation of ORO staining with human type I muscle fibers compared to lower staining in type II fibers [8]. Our data suggest at least a subpopulation of likely type II (e.g., perhaps IIa) fibers can contain a visible amount of LD in skeletal muscle taken from rodents. Overall, our data indicate that BODIPY (493/503) can be successfully used to image LDs within skeletal muscle using multiple different approaches. In addition, in much the same fashion as it is currently done for ORO staining, it would be possible to quantify LD in sections $[4,12]$ or as it was previously done in single fibers [13].

In summary, we found that BODIPY (493/503) works well as a means to visualize LD in skeletal muscle. This versatile dye works well in sectioned muscle and live culture under 


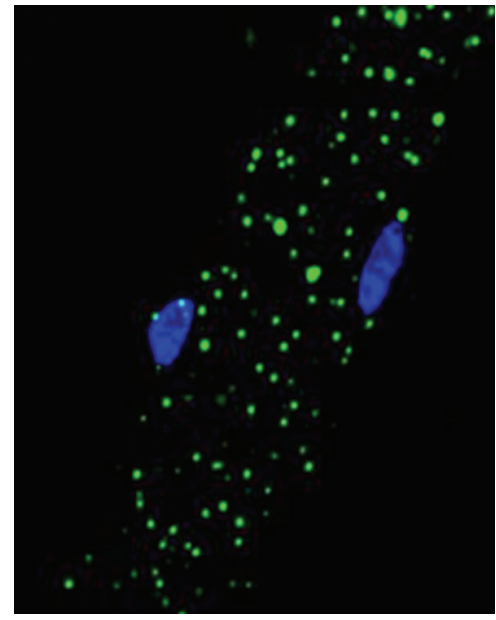

(a)

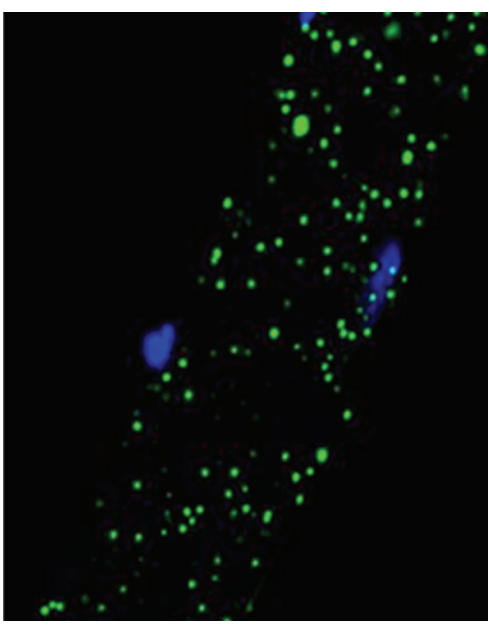

(d)

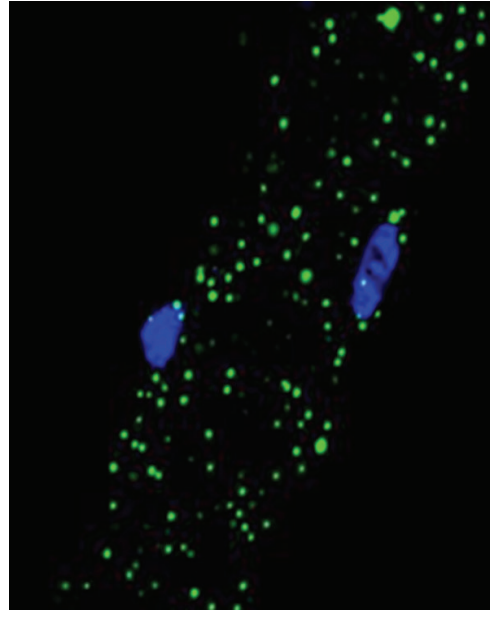

(b)

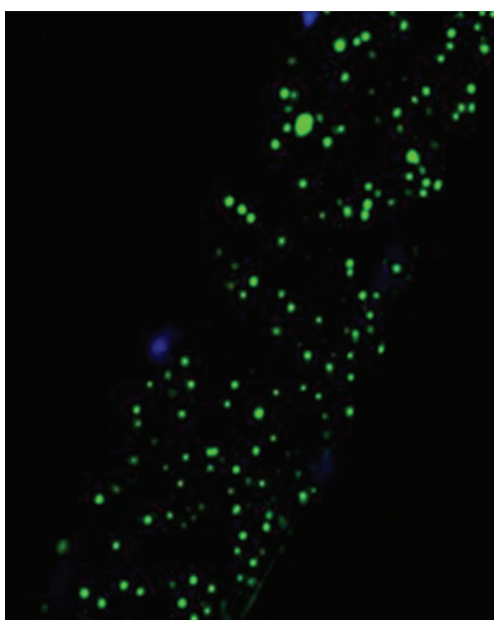

(e)

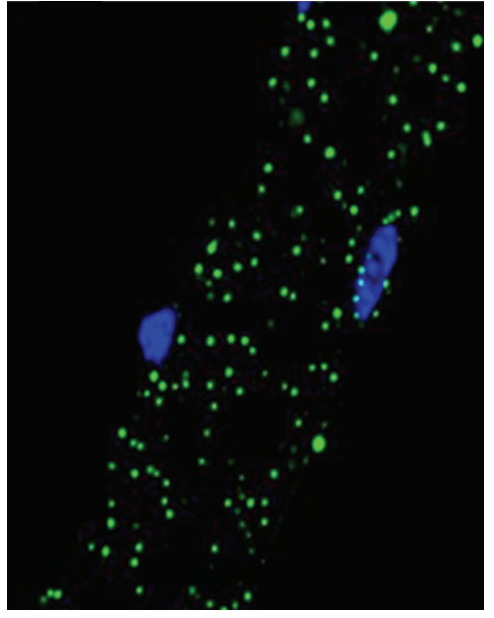

(c)

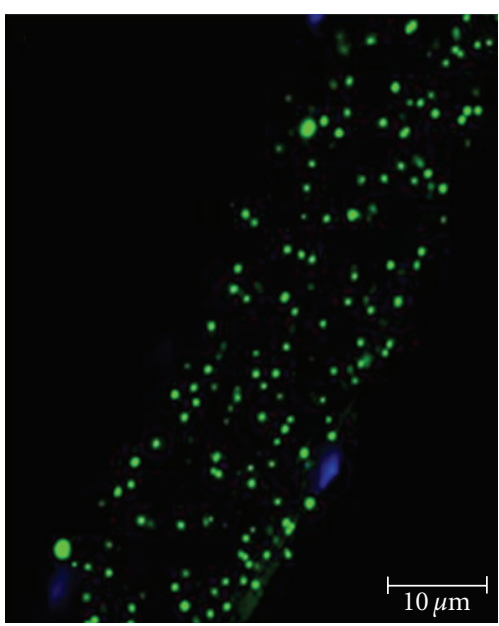

(f)

FIGURE 6: Z-stack analysis of a mouse single muscle fiber treated overnight with palmitate-oleate-supplemented media demonstrates distribution of LDs throughout the entire muscle fiber. Single muscle fibers isolated from the FDB were doublly stained with BODIPY (493/503-green) and DAPI (blue). Consecutive 1- $\mu \mathrm{m}$-thick confocal images are shown from Z-stacks taken near the middle of the fiber $(63 \mathrm{x})$, and scale bar is provided for reference.

different types of conditions. BODIPY (493/503) offers a valuable and simple alternative for investigators interested in LD function in skeletal muscle.

\section{Acknowledgment}

This work was supported by grants to R. M. Lovering from the National Institutes of Health (K01AR053235 and 1R01AR059179).

\section{References}

[1] V. B. Schrauwen-Hinderling, M. K. C. Hesselink, P. Schrauwen, and M. E. Kooi, "Intramyocellular lipid content in human skeletal muscle," Obesity, vol. 14, no. 3, pp. 357-367, 2006.
[2] L. J. C. van Loon and B. H. Goodpaster, "Increased intramuscular lipid storage in the insulin-resistant and endurancetrained state," Pflugers Archiv, vol. 451, no. 5, pp. 606-616, 2006.

[3] T. A. Wren, S. Bluml, L. Tseng-Ong, and V. Gilsanz, "Threepoint technique of fat quantification of muscle tissue as a marker of disease progression in Duchenne muscular dystrophy: preliminary study," American Journal of Roentgenology, vol. 190, no. 1, pp. W8-W12, 2008.

[4] K. De Bock, T. Dresselaers, B. Kiens, E. A. Richter, P. Van Hecke, and P. Hespel, "Evaluation of intramyocellular lipid breakdown during exercise by biochemical assay, NMR spectroscopy, and Oil Red O staining," American Journal of Physiology, vol. 293, no. 1, pp. E428-E434, 2007.

[5] E. Bonilla and A. Prelle, "Application of nile blue and nile red, two fluorescent probes, for detection of lipid droplets in human skeletal muscle," Journal of Histochemistry and Cytochemistry, vol. 35, no. 5, pp. 619-621, 1987. 
[6] C. S. Shaw, D. A. Jones, and A. J. M. Wagenmakers, "Network distribution of mitochondria and lipid droplets in human muscle fibres," Histochemistry and Cell Biology, vol. 129, no. 1, pp. 65-72, 2008.

[7] C. S. Shaw, M. Sherlock, P. M. Stewart, and A. J. M. Wagenmakers, "Adipophilin distribution and colocalisation with lipid droplets in skeletal muscle," Histochemistry and Cell Biology, vol. 131, no. 5, pp. 575-581, 2009.

[8] L. J. C. Van Loon, R. Koopman, R. Manders, W. van der Weegen, G. P. van Kranenburg, and H. A. Keizer, "Intramyocellular lipid content in type 2 diabetes patients compared with overweight sedentary men and highly trained endurance athletes," American Journal of Physiology, vol. 287, no. 3, pp. E558-E565, 2004.

[9] L. L. Listenberger and D. A. Brown, "Fluorescent detection of lipid droplets and associated proteins," Current Protocols in Cell Biology, chapter 24, unit 24 22, 2007.

[10] R. M. Lovering, L. Michaelson, and C. W. Ward, "Malformed mdx myofibers have normal cytoskeletal architecture yet altered EC coupling and stress-induced $\mathrm{Ca}^{2+}$ signaling," American Journal of Physiology, vol. 297, no. 3, pp. C571-C580, 2009.

[11] S. K. Pinnamaneni, R. J. Southgate, M. A. Febbraio, and M. J. Watt, "Stearoyl CoA desaturase 1 is elevated in obesity but protects against fatty acid-induced skeletal muscle insulin resistance in vitro," Diabetologia, vol. 49, no. 12, pp. 3027 3037, 2006.

[12] J. He, S. Watkins, and D. E. Kelley, "Skeletal muscle lipid content and oxidative enzyme activity in relation to muscle fiber type in type 2 diabetes and obesity," Diabetes, vol. 50, no. 4, pp. 817-823, 2001.

[13] C. Prats, M. Donsmark, K. Qvortrup et al., "Decrease in intramuscular lipid droplets and translocation of HSL in response to muscle contraction and epinephrine," Journal of Lipid Research, vol. 47, no. 11, pp. 2392-2399, 2006.

[14] B. A. Clark, M. Alloosh, J. W. Wenze, M. Sturek, and T. Y. Kostrominova, "Effect of diet-induced obesity and metabolic syndrome on skeletal muscles of Ossabaw miniature swine," American Journal of Physiology, vol. 300, no. 5, pp. E848-E857, 2011.

[15] T. Y. Kostrominova, "Application of WGA lectin staining for visualization of the connective tissue in skeletal muscle, bone, and ligament/tendon studies," Microscopy Research and Technique, vol. 74, no. 1, pp. 18-22, 2011.

[16] P. M. Gocze and D. A. Freeman, "Factors underlying the variability of lipid droplet fluorescence in MA-10 Leydig tumor cells," Cytometry, vol. 17, no. 2, pp. 151-158, 1994. 

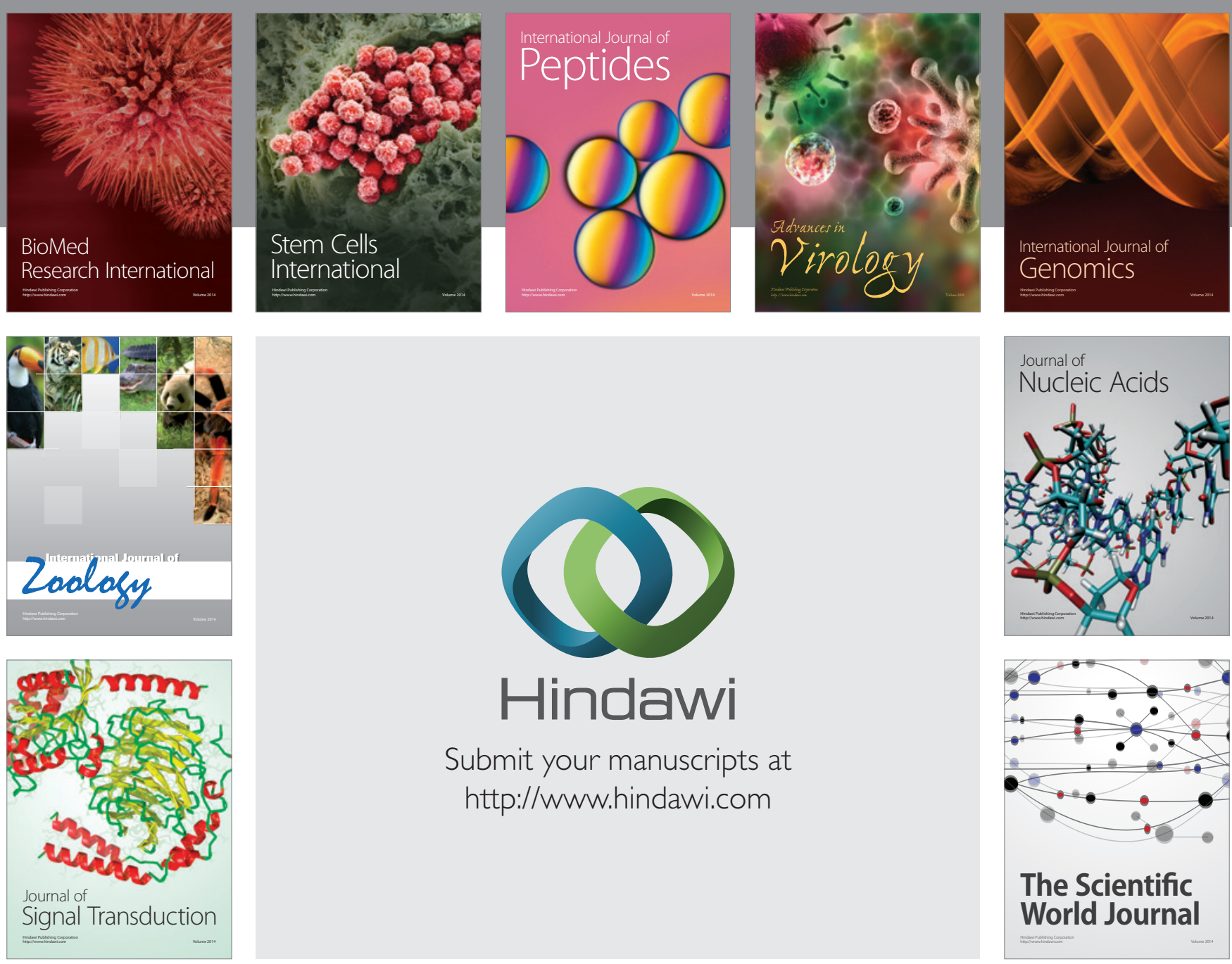

Submit your manuscripts at

http://www.hindawi.com
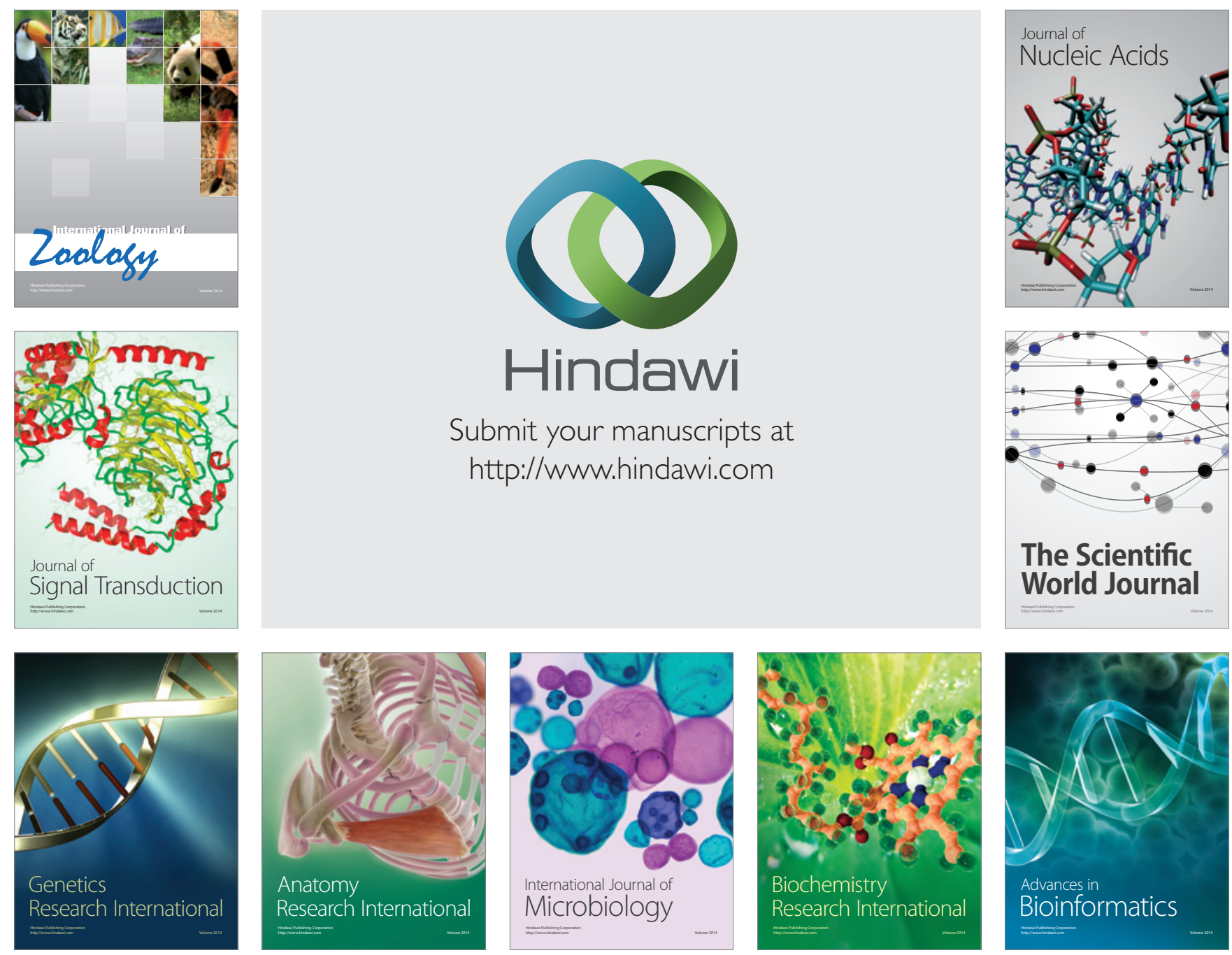

The Scientific World Journal
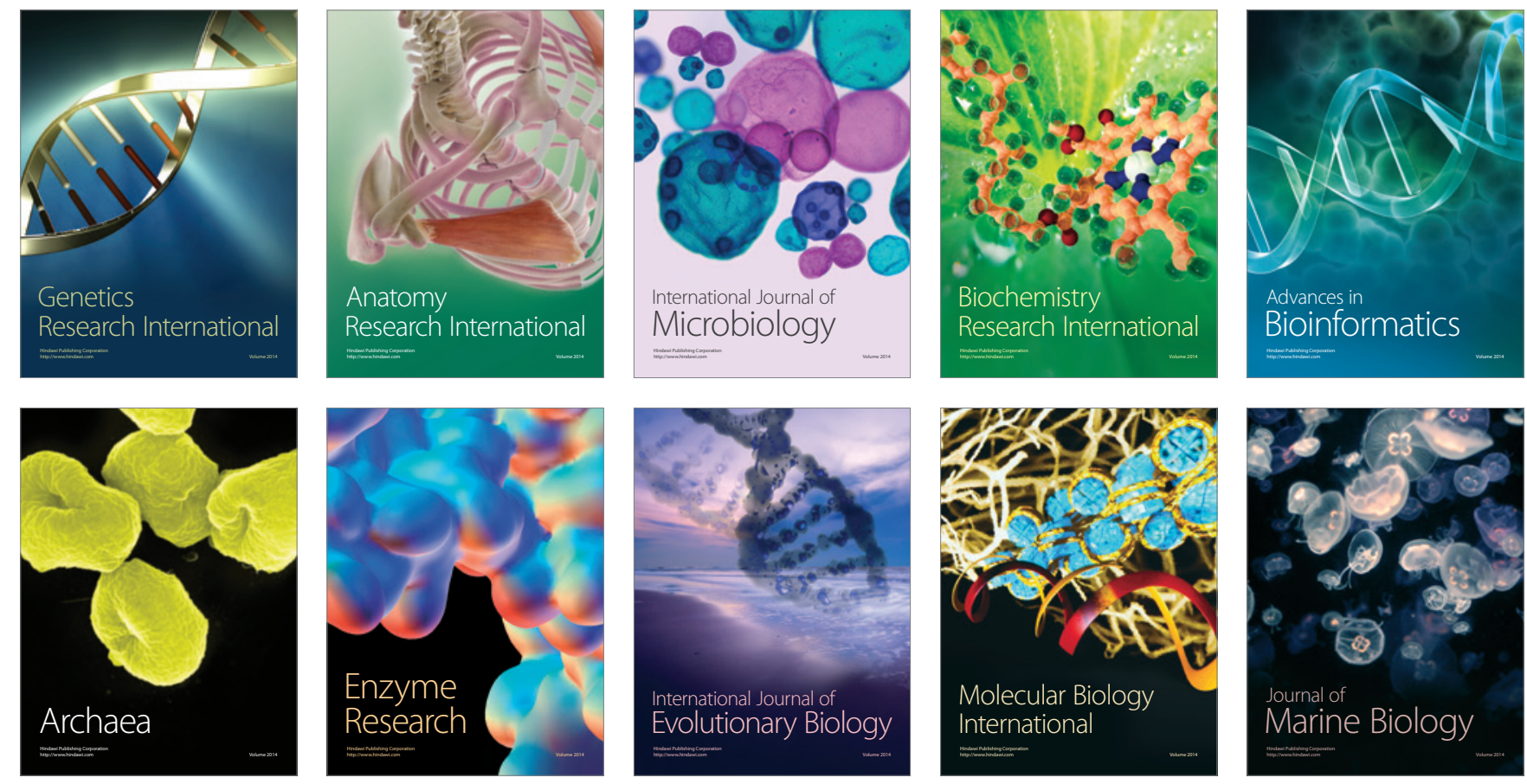